\title{
The Case for Redefinition of Frequency and ROCOF to Account for AC Power System Phase Steps
}

\author{
Andrew J. Roscoe, Adam Dyśko \\ Department of Electronic and Electrical Engineering \\ University of Strathclyde \\ Glasgow, Scotland. \\ Andrew.J.Roscoe@strath.ac.uk
}

\author{
Ben Marshall \\ National Grid, Warwick, England.
}

\begin{abstract}
All conventional techniques for measuring frequency result in large deviations to the perceived or calculated frequency when the AC waveform undergoes a phase step. The deviation magnitude and duration are dependent on the phase step magnitude, and the applied windowing/filtering. Such phase steps do occur in the power system, and the erroneous frequency calculation can result in inappropriate reactions by some rapidly-responding control and protection systems. If the frequency measurand is further differentiated to ROCOF (Rate of Change of Frequency), the excursion magnitudes can become far larger than any normally expected values of ROCOF. This paper discusses the meaning of the terms frequency and ROCOF, and presents a modified concept of frequency and ROCOF. This is done by allowing rapid phase steps to be disaggregated from frequency in the $\mathrm{AC}$ waveform model equation. This allows new measurands "underlying frequency", and "underlying ROCOF" to be defined, as a pair of linked parameters, independent from a separate dynamic phase parameter. These new measurands have the potential to offer much more useful and stable information to be sent to fast-acting control and protection systems, than the existing measurands of $A C$ frequency and ROCOF, particularly during fault events and large switching or disconnection events.
\end{abstract}

Keywords- Frequency estimation, Power System Control, Power System Faults, Power system protection, Power system reliability, Rate of Change of Frequency

\section{PHASE STEPS IN THE POWER NETWORKS}

On 16 August 2016 a major fire broke out in the Cajon Pass in southern California. It impacted several high-voltage transmission lines in the area, and caused the loss of nearly $1200 \mathrm{MW}$ of generation. Some of the generation loss was triggered by a phase jump that caused the "calculated frequency" to cross a level that required the generation (photovoltaic) to relay out.

Fig. 1, adapted from [1] shows the three phase voltage waveform, with two times at which abrupt phase shifts took place. The first of these, just before $t=0$ in the figure, occurred as a result of a phase-to-phase fault on the transmission circuit.

This work is part of the EU joint research project "Standard tests and requirements for rate-of-change of frequency (ROCOF) measurements in smart grids" which has received funding from the EMPIR programme co-financed by the Participating States and from the European Union's Horizon 2020 research and innovation programme.

\author{
Martin Lee \\ Scottish and Southern Energy Networks, Perth, Scotland. \\ Harold Kirkham \\ Pacific Northwest National Laboratory, Richland, WA, \\ USA

\section{Gert Rietveld} \\ VSL, Delft, The Netherlands.
}

The voltages of the two phases become one at the instant of the fault, and rapid change in amplitude and phase occurs, seen on the two affected phases.

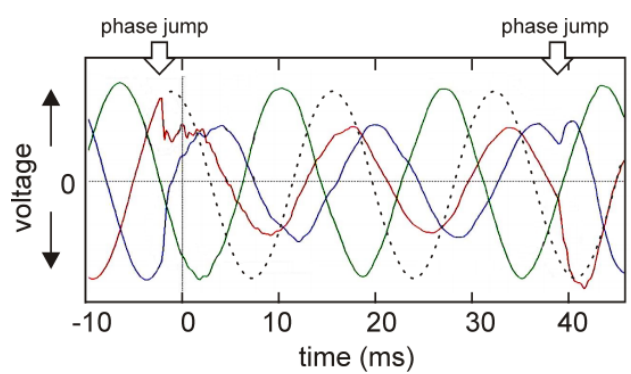

Fig. 1. Phase jumps during the August 2016 California event

The second phase jump occurs just before $\mathrm{t}=40 \mathrm{~ms}$, a little over two cycles later when the fault clears. The phase and amplitudes of the two affected phases is restored quickly to the pre-fault values. A dotted line has been added to show what the voltage on one of the phases would have been in the absence of the fault.

Similarly, within the UK, there have been several recent events which primarily involve phase (and amplitude) jumps on the power network (as opposed to genuine frequency changes), which have caused - or have threatened to cause - cascaded protection and control system actions which can lead to loss of generation and genuine frequency management problems.

For example, in a storm over the Southeast of England on a winter's evening in January 2017, multiple $400 \mathrm{kV}$ faults occurred within several minutes. Each was cleared within $150 \mathrm{~ms}$, and subsequently re-closed. No "underlying frequency" change or "underlying ROCOF" occurred. PMU (Phasor Measurement Unit) data from P-class units with 2-3 cycle measurement windows $(40-60 \mathrm{~ms})$ shows that during the events, phase steps of up to 20 degrees were observed, which were quickly reversed when the faults were cleared. An example of the phase perceived at three of the PMUs is shown in Fig. 2. 


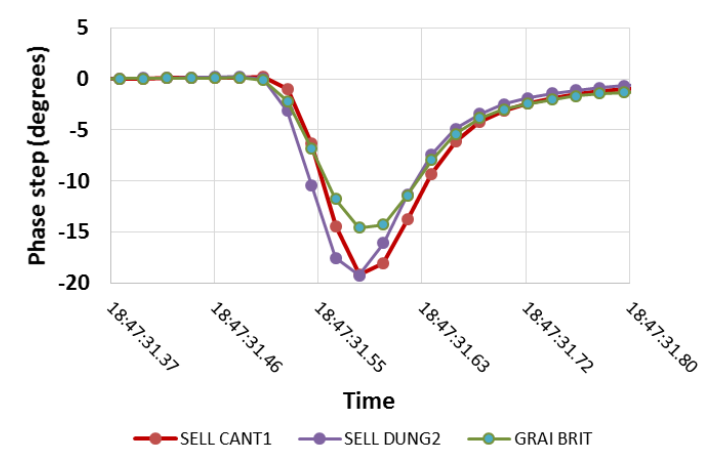

Fig. 2. Phase steps perceived by 3 PMUs during UK event in January 2017

The frequencies reported by the same three PMUs are shown in Fig. 3. There is a perceived deviation of approximately $-1 \mathrm{~Hz}$ and $+0.7 \mathrm{~Hz}$.

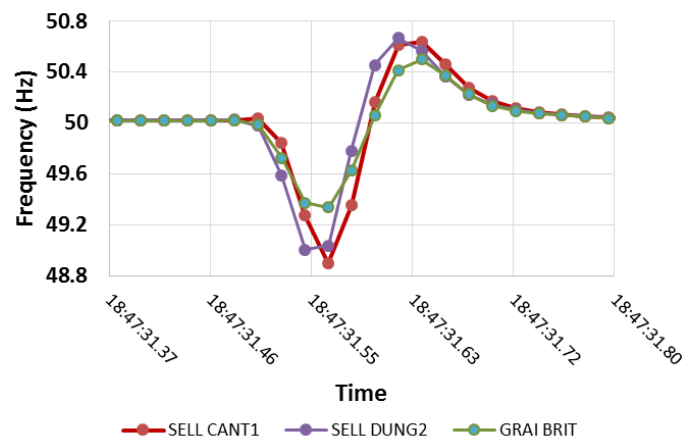

Fig. 3. Frequency perceived by 3 PMUs during UK event in January 2017

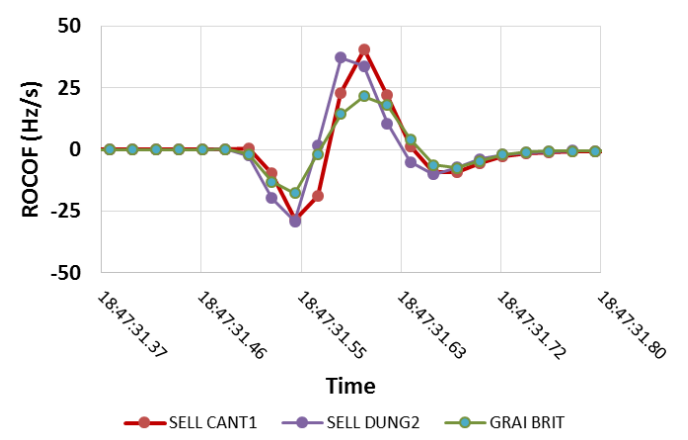

Fig. 4. ROCOF perceived by 3 PMUs during UK event in January 2017

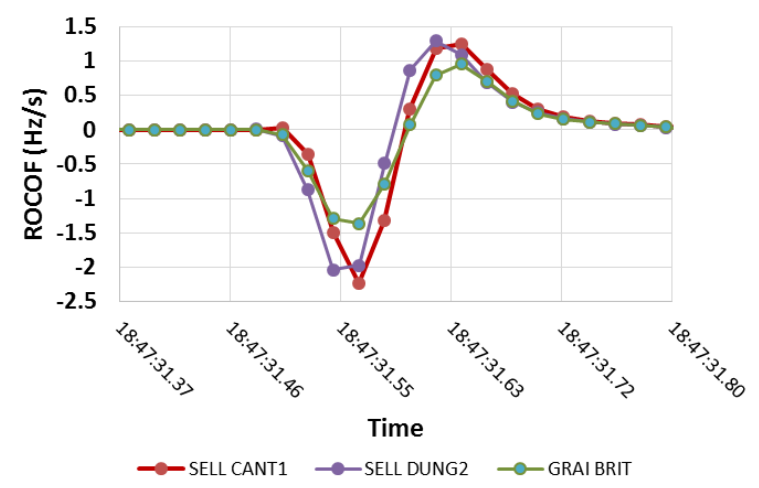

Fig. 5. ROCOF perceived by 3 PMUs during UK event in January 2017, deduced over an extended $0.5 \mathrm{~s}$ window using the finite difference of frequency
The ROCOF reported by the PMUs with such short windows shows results (Fig. 4) which are clearly well beyond the normal range of system ROCOF. Even if a $0.5 \mathrm{~s}$ post-processing window is applied to the data from Fig. 4, the perceived ROCOF is still beyond any normal bounds (typically $<0.2 \mathrm{~Hz} / \mathrm{s}$ within large power networks), as shown in Fig. 5. These traces demonstrate the threat to any relay or control system which makes a decision based on conventional frequency or ROCOF perception.

An example with a much more noticeable impact was the Langage-Landulph UK incident in May 2016 just after 11 AM . This was a single line fault (most likely lightning-induced) on one circuit of a $400 \mathrm{kV}$ line, which was cleared within $120 \mathrm{~ms}$, and successfully re-closed approximately 30 seconds later. Since parallel lower-voltage circuits remained in operation, no complete disconnection was made to supply, and the (at least initial) perception of the event to generators and measurement devices was only due to the phase (and amplitude) step in the downstream voltage waveforms. The phase step was due to the fault (for just $120 \mathrm{~ms}$ ), and an increased line impedance which persisted for $30 \mathrm{~s}$ due to the tripped line. The underlying network frequency initially remained constant and no major upstream inertia-carrying generators were tripped. However, due to the way that frequency is perceived using conventional methods, short-window devices such as P class PMUs perceived the event as shown in Fig. 6.

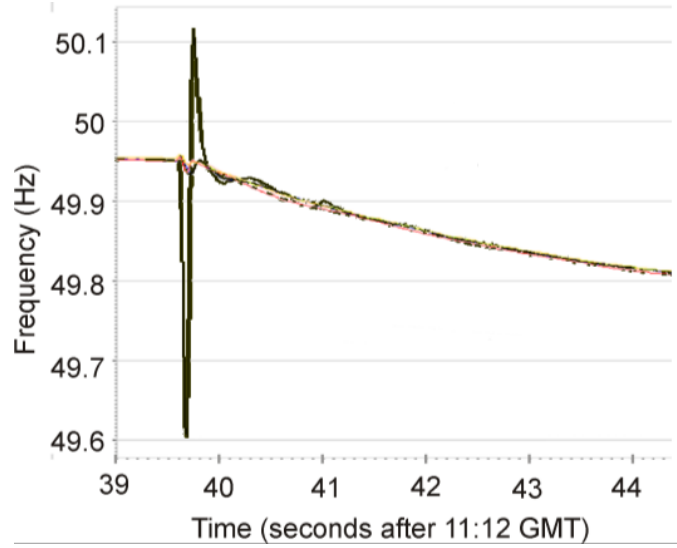

Fig. 6. Perceived frequency on May $16^{\text {th }}, 2016$, UK SW region.

\section{A. Assessment of frequency and ROCOF}

The shape of Fig. 6 at the beginning of the event, is quite similar to that of Fig. 3, and is clearly caused by a phase-step phenomenon, rather than a genuine change of "underlying frequency". In fact, the distinct phase step at the start of this event is estimated at 5-6 degrees at the PMU closest to the event, much less than the $\sim 20$ degrees registered in the January 2017 faults. However, the effect on generation was severe. National Grid estimated that 400MW of distributed PV generation was tripped, almost certainly due to a perception of loss-of-mains (LOM) made by vector-shift relays, which are commonly set at 6-12 degree thresholds. It is concluded that a number of these relays tripped, since many of them may have seen phase shifts at or above the 6 degree level. Essentially they functioned correctly - they are designed to detect a phase step, and a phase step they did see. However, a phase step of 20 degrees (or more) 
can clearly occur when there is no reason to trip a generator. This has led to the general recommendation to stop using vector-shift relays [2]. This is relevant because a vector-shift relay is essentially performing a similar function to a ROCOF-detection relay, over a short window of just a few cycles (or even $<1$ cycle).

All this evidence tells us that rapid phase steps do happen on our power networks, at up to (and perhaps more than) 20 degrees. It also suggests that existing measurement devices perceive the phase steps not only as the steps themselves, but as large frequency and ROCOF deviations. The magnitude of the perceived frequency and ROCOF deviations can be reduced by using longer time windows (more filtering), but this also dilates the deviation in time. Long time windows and high ROCOF values may be acceptable in some applications. For example, within both Ireland and the UK, the recommended settings for anti-islanding (Loss of Mains, LOM) relays, have been increased from $0.125-0.25 \mathrm{~Hz} / \mathrm{s}$ to $1 \mathrm{~Hz} / \mathrm{s}$, assessed over a relatively long $0.5 \mathrm{~s}$ window [3]. Nevertheless, Fig. 5 shows that even such long windows may not offer a full solution.

Additionally, there are many applications which are time sensitive, which forces the use of a relatively short window, and which require accurate and stable estimates of frequency and ROCOF. A typical example would the measurement of frequency or ROCOF to dispatch active power in any "fast frequency response" mechanism. The ongoing project "Enhanced Frequency Control Capability" [4, 5], requires a full active-power response from participating devices, within $0.5 \mathrm{~s}$ of the initial event. This means that measurement, communication \& aggregation, and controlled active-power injection, must all occur within 500 ms. The measurement must therefore account for only a fraction of the allocated time. While the time latency of the measurement is technically only half the window length, the actual measurement latency is longer due to numerical computation of the window/filter output, plus the latency required to extract and communicate the digital measurements from the measuring device. This means that to have a closed-loop active-power control system, using frequency or ROCOF as the input variable, the frequency and ROCOF assessment window needs to be significantly less than $500 \mathrm{~ms}$. In such a use case, it is crucial to find some way of avoiding the "wild" control signals which will result from short time-window measurements like Fig. 3, Fig. 4, and Fig. 6. A "wild and crazy" value of frequency or ROCOF, which bears no useful relationship with the underlying synchronized area frequency and ROCOF, perceived for even a short time like 100$200 \mathrm{~ms}$, can wreak havoc within a control system.

\section{B. A question of signal models}

The crux of this paper lies in appreciating that there are different signal models which can be applied to the same actual voltage waveform. Then, it is possible to appreciate that some of the confusion concerning perceived frequency and ROCOF behaviour stems from the fact that the user may be applying (perhaps accidentally/unconsciously) one signal model concept, while the measured voltage values are a poor fit to that model [6]. This can cause many problems, especially when phase jumps are considered [7].

\section{HOW FREQUENCY IS CONVENTIONALLY MEASURED}

\section{A. The relative lack of standardisation of algorithms}

There is a remarkable freedom for manufacturers of different devices to implement all manner of different algorithms and mechanisms to assess fundamental frequency. Power quality standards such as IEC 61000-4-30 suggest 10 second (or longer) windows, but allow shorter windows such as 10/12 cycles, and do not mandate, nor suggest, any particular method [8]. There likewise is no standard which governs the window lengths/shapes, or the methods or algorithms, by which class 81 relays assess frequency, nor by which class $81 \mathrm{R}$ relays assess ROCOF. An evolving standard exists for PMUs [9, 10] which will also emerge in a slightly updated form as IEC 60255-1181. While frequency and ROCOF assessment was never the prime motivation for developing PMU technology, this standard has ended up being the only common reference for devices which assess frequency and ROCOF over the shorter time windows relevant to this paper.

While the existing PMU standard does not come close to testing a device against all scenarios it will meet in the real world, there are tests which apply various dynamic events, including $10^{\circ}$ phase steps of the AC waveform $[9,10]$. These phase steps are not dissimilar to some of those encountered during real-world dynamic network events, although of course real events can be much more complex involving unbalance and other periodic and aperiodic oscillations.

A key observation of the PMU test procedure is that during phase steps, a response time is defined, which is essentially a time window, commensurate with the time window used by the measurement device, that straddles the time of the instantaneous applied phase step. When assessing amplitude and phase perceptions from the PMU, it is expected that the measurands track in a roughly linear manner from the pre-event values to the post-event values, over the course of the response time. However, the frequency and ROCOF outputs from the PMU are allowed to vary during the response time, bearing almost no relation to the pre-event or post event frequency and ROCOF values. So long as the values return to an accurate state after the response time has expired, the device is considered to be compliant. This is not a specific of PMUs nor of the PMU standard. It is a simple matter that the term "frequency" is not well defined for signals with such steps [11]. All conventional devices measuring frequency and ROCOF will likely respond with similarly perturbed outputs during the phase step scenario, as was shown in section I.

\section{B. The conventional signal model used for Fourier-based measurements}

When a conventional PMU, or any device using a Fourier technique to determine the amplitude and phase of an $\mathrm{AC}$ waveform is used, the device first forms a measurement estimate of amplitude and phase of the fundamental over a time window, which is defined by the filtering (shaped time windowing) applied. Essentially, the Fourier analysis process allows the voltage magnitude $X_{M}(t)$ and the phase $\theta(t)$ to be estimated from the voltage waveform samples $x(t)$. The signal model which is being applied for the fundamental signal is: 


$$
x(t)=X_{m}(t) \cos (\theta(t))
$$

If highly-overlapping, continuously rolling windows are used, it is possible to evaluate a new estimate of $X_{M}(t)$ and $\theta(t)$ for every new sample input $x(t)$. This is highly desirable within local converter control schemes which update at several $\mathrm{kHz}$ switching speed. Alternatively, decimated estimates of the parameters can be produced by using windows which overlap less, or do not overlap at all. The estimates always have a latency which is half the window length, assuming a symmetric window is used, and in dynamic conditions the values are affected by the window length and shape.

Based on (1), the conventional approach is to define frequency as the rate-of-change of phase (divided by $2 \pi$ ), and ROCOF as the rate-of-change of frequency. Hence, considering the actual measurement process where windowed and estimated quantities are involved:

\begin{tabular}{|l|l|}
\hline$f_{W}(t)=\frac{1}{2 \pi} \cdot \frac{d \theta_{W}(t)}{d t}$ & (2) \\
\hline $\operatorname{ROCOF}_{W}(t)=\frac{d f_{W}(t)}{d t}$ & (3) \\
\hline
\end{tabular}

where $f_{W}(t)$ and $\operatorname{ROCOF}_{W}(t)$ are the windowed estimates.

Optionally, additional filtering or time-windowing is applied in the determination of frequency from phase, either intentionally, or un-intentionally due to use of a (potentially down-sampled) finite-difference techniques. The same is true for the second differentiation step from frequency to ROCOF. This means that the combined windowing effect on the ROCOF measurement can be a complex cascade of 3 windowed steps.

In terms of the mathematical model which the measurements are estimating, the equations are identical except that the windowing complexities can be overlooked:

\begin{tabular}{|l|l|}
\hline$f(t)=\frac{1}{2 \pi} \cdot \frac{d \theta(t)}{d t}$ & (4) \\
\hline$R O C O F(t)=\frac{d f(t)}{d t}$ & (5) \\
\hline
\end{tabular}

where $f(t)$ and $\operatorname{ROCOF}(t)$ are the parameters in the ideal signal model.

This signal model allows the signal phase to be defined by either the phase trajectory directly, or the frequency, or the ROCOF. All three of these parameters are linked and wholly dependent according to (4) \& (5). Defining any one of the triplet defines the other two. Essentially there is only one "freely variable" parameter, but it can be expressed in three different forms. For example, a constant-ROCOF frequency ramp at $R \mathrm{~Hz} / \mathrm{s}$ for over time $t_{1}$ to $t$ is most easily defined in the signal model by fixing the single parameter $\operatorname{ROCOF}(t)=R$. This then directly defines $f\left(t_{0}\right)$ and $\theta\left(t_{0}\right)$ :

\begin{tabular}{|l|}
$f(t)=\int_{\tau=t_{1}}^{t} R \cdot d \tau+f\left(t_{1}\right)$ \\
$\Rightarrow f(t)=[R \tau]_{t_{1}}^{t}+f\left(t_{1}\right)$ \\
$\Rightarrow f(t)=R\left(t-t_{1}\right)+f\left(t_{1}\right)$
\end{tabular}

and:

$$
\begin{gathered}
\theta(t)=\int_{\tau=t_{1}}^{t} 2 \pi f(\tau) \cdot d \tau+\theta\left(t_{1}\right) \\
\Rightarrow \theta(t)=\left[\pi R \tau^{2}\right]_{t_{1}}^{t}-2 \pi R t_{1}\left(t-t_{1}\right) \\
\quad+f\left(t_{1}\right)\left(t-t_{1}\right)+\theta\left(t_{0}-T\right) \\
\Rightarrow \theta(t)=\pi R\left(t-t_{1}\right)^{2}+f\left(t_{1}\right)\left(t-t_{1}\right)+\theta\left(t_{1}\right)
\end{gathered}
$$

This describes the parabolic trajectory of phase during a genuine constant-ROCOF event, explaining how the average phase across a time window is not the same as the phase at the centre of the window [12]. PMU applications need to be especially careful of this phenomenon!

The important point we want to make here for this paper is that we could alternatively define the same constant-ROCOF event by either (6) or (7). Any one of the three variables in the triplet defines the other two in this signal model.

The conventional signal model for measurement can be slightly adapted, as is done for PMU measurements, to make the reported synchrophasor phase $\phi$ relative to a reference waveform at nominal frequency $f_{0}$, rather than simply the point-on-wave phase $\theta$ of the cosine wave[9]. This can be done by defining a phase parameter $\phi(t)$ as:

$$
\phi(t)=\theta(t)-2 \pi f_{0} t
$$

If it is desired to build the signal model as a function of $\phi$, then equations (2) \& (4) simply need to be adjusted by $f_{0}$, while (3) \& (5) remain unchanged. For example (4) becomes:

$$
\begin{array}{|l|l}
\hline f(t)=\frac{1}{2 \pi} \cdot \frac{d \phi(t)}{d t}+f_{0} & \text { (9) }
\end{array}
$$

\section{Conventional use of zero crossings techniques}

The time between zero crossings is determined by the time taken for $\theta$ to increment by $2 \pi$, and so the measured frequency from an algorithm using zero-crossings is still directly determined by the phase trajectory. Therefore, in a zero-crossing measurement, the triplet of phase, frequency and ROCOF are still entirely dependent and linked as in (4) \& (5).

\section{Non-conventional methods using similar signal models}

There are many algorithms based on techniques other than Fourier analysis or zero-crossings, which have been proposed as options to provide PMU phasor-measurement functionality, and also estimates of frequency and phase as bi-products. Some of these initially appear to provide signal models which allow phase to be assessed independently from frequency (and ROCOF). However, usually the end result of the whole algorithm is to provide frequency (and ROCOF) results which are linked to the phase estimation, in the same linked triplet. 
For example,in the Prony method reported in [13] (1)-(2), the signal model seems to estimate phase and frequency separately. However, the algorithm actually requires the estimate of frequency first, and assesses amplitude and phase as a "residual", through which the frequency can be determined from phase directly via (2), and then fed back. Similarly, methods based on a "Least Squares" analysis [14] [15] apply a simpler waveform model in which the dynamic phasor (amplitude and phase) is estimated, and then frequency and ROCOF are determined from the trajectory of the phasor.

\section{E. A phase step using the conventional signal model}

A phase step can be defined in the conventional model. Consider first the ideal signal model given by (1), (4) \& (5). A phase step of $\Delta$ radians at time $t_{\mathrm{S}}$, superimposed on an underlying stable frequency $f_{0}$, with underlying $\mathrm{ROCOF}=0$ could be expressed as a Heaviside (step) function, with the terminology that $H(t)$ is a unit magnitude step from 0 to 1 at $t=0$ :

$$
\begin{aligned}
& \theta(t)=\int_{t_{1}}^{t} 2 \pi f(\tau) \cdot d \tau+\theta\left(t_{1}\right)+\Delta \cdot H\left(t-t_{S}\right) \\
& \Rightarrow \theta(t)=2 \pi f_{0}\left(t-t_{1}\right)+\theta\left(t_{1}\right)+\Delta \cdot H\left(t-t_{S}\right)
\end{aligned}
$$

However, when the frequency of this ideal signal is deduced, by (4), it requires the use of a delta-function with infinite height and infinitely short time, and weight $\Delta / 2 \pi$ :

$$
f(t)=f_{0}+\frac{\Delta}{2 \pi} \cdot \delta\left(t-t_{S}\right)
$$

Due to the impulse, ROCOF cannot be deduced using (5) in any rational way, as the differential of a delta-function is undefined. Thus, the ideal version of the conventional model used to measure power system signals is theoretically incapable of dealing with phase steps!

In reality, any real measurement system will always apply some finite time windowing, and so the frequency perceived for a phase step is a filtered version of a delta-function, with finite deviation magnitude and a dilated time over which the deviation occurs. This is exactly what is seen in Fig. 3 and Fig. 6. For example, if the underlying frequency is $f_{0}$, and the phase step occurs:

$$
\Delta=2 \pi \int\left(f_{W}(t)-f_{0}\right) \cdot d t \quad \text { over the window } T_{\mathrm{W}}
$$

where $f_{W}(t)$ is the perceived frequency from the windowed output.

For example, with a 10 degree $(0.17 \mathrm{rad})$ phase step, and a 5 -cycle $(100 \mathrm{~ms})$ window, the average perceived frequency deviation is $0.27 \mathrm{~Hz}$, from:

$$
\overline{\left(f_{W}(t)-f_{0}\right)}=\frac{\Delta}{2 \pi T_{W}}, \quad T_{W}=\text { window time }
$$

This is a significant deviation! The peak deviation can be much larger if the window is not rectangular, but has a more complex centre-weighted shape, such as the filters commonly used to obtain better frequency-domain performance. The perceived ROCOF in reality is a differentiated version of a filtered/windowed delta function, typically like Fig. 4 or Fig. 5.

The raises the question; is (1), (4) \& (5) the most useful signal model to use, and are those "definitions" of frequency and ROCOF the most useful measurands to use for protection and control systems? The next section proposes an alternative model, which leads to more useful measurands of "underlying frequency" and "underlying ROCOF", without the need to resort to long window lengths.

\section{AN ALTERNATIVE SIGNAL MODEL}

In contrast to the previous section, it is shown here how it is possible to define a different mathematical model which allows new measurands "underlying frequency" and "underlying ROCOF" (as a linked pair) to be partly disaggregated from phase, by also splitting phase into two disaggregated components.

The signal model could be re-expressed as:

\begin{tabular}{|l|c|}
\hline$x(t)=X_{m}(t) \cos (\theta(t)+\psi(t))$ & $(14)$ \\
\hline \multicolumn{1}{|c|}{ In (14) the phase argument has been split out into two }
\end{tabular}
independent parameters. $\theta$ is a phase component which relates to "underlying frequency" and "underlying ROCOF", as an interlinked pair, and includes slow (band-limited) deviations in phase offset which do not relate to significant phase steps. The "underlying frequency" and "underlying ROCOF" relate to $\theta$ in exactly the same manner as the conventional model, via (4) \& (5). The new phase parameter is $\psi(t)$. This contains filtered elements of phase offset which represent significant phase steps. Essentially, $[\theta(t)+\psi(t)]$ in (14) is equivalent and equal to $\theta(t)$ in (1), with $\psi(t)$ containing information for the rapid phase transients, and $\theta(t)$ defining all the other components of phase. Underlying frequency and ROCOF are assessesed only on the basis of $\theta$, and wholly independent of $\psi$. Assessment of the phase measurand, for PMU purposes, would still be based on $[\theta(t)+\psi(t)]$ and include both components of phase.

This alternative model allows rapid phase steps to be modelled in $\psi(t)$ using either Heaviside functions, or Heaviside functions filtered with short time-length filters. Essentially, during any rapid phase step, $\psi(t)$ can be used to express the rapid phase transition, while $\theta(t)$ can remain relatively unperturbed, defined by the underlying $f(t)$ which can remain nearly constant, ROCOF which can remain nearly zero, and the slower deviations in phase offset. It is interesting to note that in the IEEE PMU standard [9], an equivalent equation to (14) is used, although it is no longer referred to in the emerging IEC 60255118 draft.

Anyone carrying out the phase step test $[9,10]$ will find that they must use a model of the form of (14) to accurately generate or analyse the test signal waveforms [16]. Otherwise, using (1), the problem of delta-functions and infinities described in section II $\mathrm{E}$ is encountered. This is a good argument to move to a more appropriate signal model such as (14).

\section{APPLYING THE ALTERNATIVE MODEL TO MEASUREMENTS}

While generating a signal waveform $x(t)$ from (14) is easy, carrying out the reverse process and disaggregating $\theta(t)$ and $\psi(t)$ 
from each other, in an effective way in real time and with minimal latency, is a significant challenge. Investigations will be done as part of the joint EU research project "Standard tests and requirements for rate-of-change of frequency (ROCOF) measurements in smart grids" [17].

The key is probably to have a short-window measurement (with time length $T_{S}$ perhaps 1-2 cycles) running on the most recent sampled data, to identify the phase-step events in $\psi(t)$. Meanwhile, a longer window measurement (time $T_{L}$, of perhaps 5-10 cycles) can be used to determine the underlying frequency and ROCOF from $\theta(t)$, after the effects of $\psi(t)$ have been "backed out", and with good rejection of out-of-band noise, flicker, and interharmonics.

To achieve the best disaggregation of $\psi(t)$ and $\theta(t)$, it may be necessary to have the longer window use only data from the region $-\left(T_{L}+T_{S}\right)<t<-T_{S}$ so that $\psi(t)$ and $\theta(t)$ can be assessed using uncorrelated data. This also allows the step to be identified (and dealt with) in the first cycle or two of real time, before it impacts on the long-window measurement. There are implications for latency, which needs to be carefully managed. It is advantageous to have the latency reasonably constant, i.e. not changing suddenly. A latency which changes suddenly can itself cause problems within dynamic control systems.

In [18], a method was presented which detects faults and phase-steps and then removes the "bad data" from the main measurement window. Such a method is worth considering, although there are some variable-latency issues to consider.

\section{CONCLUSIONS}

The key conclusion of this paper is that a new signal model may provide a much better definition of the measurands for "underlying frequency" and "underlying ROCOF" than the existing signal models, by disaggregating the signal model phase into two parts. One part is a high-bandwidth dynamic phase term which contains information that pertains to rapid or step phase changes. The other part contains phase information that corresponds to the more steadily-progressing phase trajectory associated with slowly-changing fundamental frequency, genuine system ROCOF, and lower-bandwidth phase offsets. While the conventional model ties together phase, frequency and ROCOF as a linked triplet of dependent parameters, the proposed model splits apart the dynamic phase parameter from the frequency and ROCOF parameters, and the low-bandwidth phase offset. The new "underlying frequency" and "underlying ROCOF" parameters are still a linked dependent pair, but the dynamic phase parameter is independent and can be used to describe phase steps.

Further research is needed on how to determine the parameters (measurands) of this alternative model from actual real-time AC sampled voltage measurements. There remains a challenge to develop the filtering or detection techniques which can effectively and reliably discriminate between the rapid phase-step information in $\psi(t)$, and the lower-bandwidth information in $\theta(t)$ which is tied to the measurands of "underlying frequency", and "underlying ROCOF" which the user would like to see remain unperturbed during phase steps.

\section{REFERENCES}

[1] NERC, "North American Electric Reliability Council 1,200 MW Fault Induced Solar Photovoltaic Resource Interruption Disturbance Report," 2017 Available:

http://www.nerc.com/pa/rrm/ea/1200 MW Fault Induced Solar Photo voltaic Resource $/ 1200$ MW Fault Induced Solar Photovoltaic Reso urce Interruption_Final.p̄edf, accessed June $20 \overline{1} 7$.

[2] "Rate of Change of Frequency protection changes to deal with increasing system Rate of Change of Frequency due to reduced system inertia and larger maximum loss of infeed (1800MW from 1320MW).",

DCRP_17_02_xx_RoCoF, Distribution Code Review Panel Meeting 63 - Thursday 8 March 2017. , 2017 Available:

www2.nationalgrid.com/WorkArea/DownloadAsset.aspx $2 \mathrm{id}=858994037$ 2, accessed June 2017.

[3] National Grid, "GC0035 / GC0079 Frequency Changes during Large Disturbances and their effect on the total system - Phase $1 \& 2$," 2016 Available: http://www2.nationalgrid.com/UK/Industryinformation/Electricity-codes/Grid-code/Modifications/GC0035GC0079/, accessed June 2017.

[4] "Enhanced Frequency Control Capability (EFCC)," 2016 Available: http://www.nationalgridconnecting.com/The balance of power/, accessed June 2017.

[5] P. Wall, N. Shams, V. Terzija, V. Hamidi, C. Grant, et al., "Smart frequency control for the future GB power system," in IEEE Innovative Smart Grid Technologies Europe (ISGT-Europe) 2016.

[6] A. Riepnieks and H. Kirkham, "An Introduction to Goodness of Fit for PMU Parameter Estimation," IEEE Transactions on Power Delivery, In Press.

[7] A. Riepnieks, H. Kirkham, A. J. Faris, and M. Engels, "Phase jumps in PMU signal generators," in IEEE PES General Meeting, Chicago, USA, 2017.

[8] IEC, "Electromagnetic compatibility (EMC) - Part 4-30: Testing and measurement techniques - Power quality measurement methods," IEC61000-4-30, 2003

[9] IEEE, "IEEE Standard for Synchrophasor Measurements for Power Systems," C37.118.1-2011, 2011.

[10] IEEE, "IEEE Standard for Synchrophasor Measurements for Power Systems -- Amendment 1: Modification of Selected Performance Requirements " C37.118.1a-2014, 2014.

[11] H. Kirkham and A. Riepnieks, "Students' simple method for determining the parameters of an AC signal," in 57th Conference on Power and Electrical Engineering, Riga, Latvia, 2016.

[12] A. J. Roscoe, I. F. Abdulhadi, and G. M. Burt, "P and M Class Phasor Measurement Unit Algorithms using Adaptive Cascaded Filters," IEEE Transactions on Power Delivery, vol. 28, pp. 1447-1459, 2013.

[13] J. A. de la O Serna, "Synchrophasor Estimation using Prony's Method," IEEE Transactions on Instrumentation and Measurement, vol. 62, pp. 2119-2128, 2013.

[14] D. Belega and D. Petri, "A Real-Valued Taylor Weighted Least Squares Synchrophasor Estimator," in IEEE Applied Measurements for Power Systems (AMPS), Aachen, Germany, 2014.

[15] D. Belega, D. Fontanelli, and D. Petri, "Low-Complexity Least-Squares Dynamic Synchrophasor Estimation Based on the Discrete Fourier Transform," IEEE Transactions on Instrumentation and Measurement, vol. 64, pp. 3284-3296, Dec 2015.

[16] D. C. Guglielmo Frigo, Asja Dervi־skadi'c, Marco Pignati, Claudio Narduzzi, Mario Paolone, "Definition of Accurate Reference Synchrophasors for Static and Dynamic Characterization of PMUs," IEEE Transactions on Instrumentation and Measurement, In Press.

[17] RocofMetrology.EU, "Standard tests and requirements for rate-ofchange of frequency (ROCOF) measurements in smart grids," 2016 Available: http://www.rocofmetrology.eu/, accessed June 2017.

[18] Z. Y. He, L. Fu, W. Z. Han, R. K. Mai, and Z. Y. Dong, "Precise algorithm for frequency estimation under dynamic and step-change conditions," IET Science Measurement \& Technology, vol. 9, pp. 842851 , Oct 2015. 